\title{
PROGRAMA ORIENTADO A DAR CONOCIMIENTO SOBRE LA PREVENCIÓN DEL SÍNDROME DEL TÚNEL CARPIANO EN ESTUDIANTES DE ODONTOLOGÍA DE I A X SEMESTRE DE LA FACULTAD DE ODONTOLOGÍA DE LA UNIVERSIDAD SANTO TOMÁS
}

\author{
${ }^{1}$ Leidy Álvarez C., ${ }^{1}$ Leslie Cañas Uribe, ${ }^{1}$ Jennifer Villamizar J., ${ }^{2}$ Gloria Cristina Aránzazu M. \\ ${ }^{1}$ Estudiante de X semestre, F. de Odontología U. Santo Tomás. \\ 2 Odontóloga U. Santo Tomás, Especialista en Patología Oral y Medios Diagnóstico U. El Bosque, Docente U. Santo Tomás.
}

Autor responsable de correspondencia: Gloria Cristina Aránzazu Moya

Dirección de correo electrónico: aranzazugloria@yahoo.es

\section{RESUMEN}

Objetivo: Desarrollar un programa orientado a prevenir el Síndrome del Túnel Carpiano (STC) en los estudiantes de odontología de la Universidad Santo Tomás, en Bucaramanga.

Materiales y métodos: Se realizó un estudio experimental del tipo ensayo clínico controlado en 105 estudiantes. Se seleccionaron dos grupos al azar, en ambos se recolecto información sobre el conocimiento de STC antes y después de la intervención. Al grupo intervenido se dio una charla con un material educativo. La selección de la muestra se hizo mediante un muestreo no probabilístico por cuotas, para el plan de análisis se consideró el test de $\mathrm{chi}^{2}$, test de rangos de Wilcoxon según la naturaleza y distribución de las variables.

Resultados: Se encontraron cambios estadísticamente significativos en cuanto a edad $(\mathrm{P}=<0.0001)$, género $(\mathrm{P}=0.868)$ y sobre el nivel de conocimiento antes y después de la intervención $(\mathrm{P}=<0.0001)$. Se obtuvo un $83 \%$ (43) de nivel de conocimiento alto en el grupo de estudiantes intervenidos y un $83 \%$ (44) nivel de conocimiento medio en los no intervenidos, después de realizar la intervención. Conclusiones: Se observó que un programa orientado a prevenir el STC es una buena estrategia comunicativa y preventiva que permite aumentar el nivel de conocimiento de manera favorable y mejorar las posibilidades de aprendizaje en el estudiante. [Álvarez L, Cañas L, Villamizar J, Aránzazu GC. Programa orientado a dar conociiento sobre la prevención del Síndrome del Túnel Carpiano en estudiantes de odontología de I a X semestre de la Facultad de Odontología de la Universidad Santo Tomás. Ustasalud Odontología 2008; 7: $42-48]$

Palabras clave: Síndrome del túnel carpiano. Estudiantes de odontología, Salud ocupacional.

\section{PROGRAM ORIENTED TO PREVENT CARPAL TUNNEL SYNDROME IN DENTAL STUDENTS FROM I TO X SEMESTER IN THE SCHOOL OF DENTISTRY OF THE SANTO TOMAS UNIVERSITY}

\section{ABSTRACT}

Objective: To develop a program oriented to avoid carpal tunnel syndrome (CTS) in dental students from Santo Tomas University, in the city of Bucaramanga.

Materials and methods: A controlled clinical essay was carried out on 105 dental students. The selection of the sample was done by means of a sampling not probabilistic by quotas, for the plan of analysis it was considered to apply the test of chi², test of ranges of Wilcoxon according to the nature and distribution of the variables.

Results: There were statistically significant changes for age $(\mathrm{P}=<0.0001)$, gender $(\mathrm{P}=0.868)$ and on the level of knowledge before and after the intervention $(\mathrm{P}=<0.0001)$.

Conclusions: It was observed that a program to prevent CTS is a good communicative and strategy that allows to increase the level of knowledge to improve the possibilities of learning in the student.

Key words: Carpal tunnel syndrome, Dental students, Occupational health. 


\section{INTRODUCCIÓN}

El túnel carpiano es una estructura anatómica en la muñeca formada por ligamentos y huesos. El nervio mediano, que lleva impulsos del cerebro a la mano, pasa por el túnel carpiano junto con los tendones que permiten cerrar la mano. Cuando se tensionan, los tendones se inflaman dentro del túnel y comprimen el nervio mediano. Además de los movimientos repetitivos, otras condiciones pueden llevar a la compresión del nervio mediano: artritis, diabetes, retención de líquido, gota y fracturas mal alineadas, desbalances químicos, tensión emocional, y a veces cambios hormonales en las mujeres. ${ }^{1}$

El Síndrome del Túnel Carpiano (STC) está relacionado con diferentes profesiones que combinan el uso de fuerza y la repetición de movimientos con los dedos y las manos; la actividad física y las malas posturas afectan a los profesionales de todas las áreas (constructores, digitadores, jardineros, empacadores) siendo los odontólogos uno de los grupos más afectados. Alrededor del $1 \%$ de las personas que tienen el STC pueden desarrollar una lesión permanente de la mano afectada.

Se dispone de poca información sobre programas orientados a la prevención del STC en odontólogos; se encontraron algunos reportes por administradores de riesgos profesionales y programas fisioterapéuticos en empresas de costura y avícolas, y algunas estrategias de acción desde el punto preventivo. ${ }^{2,3}$

Sin embargo, los informes de lesiones por movimientos repetitivos en los lugares de trabajo se han incrementado dramáticamente en los últimos años. Cerca del $60 \%$ de todas las enfermedades ocupacionales ocurren por desordenes de trauma repetitivo. Adicionalmente, esta es la neuropatía más común y tiene una alta prevalencia en la población general. En Minnesota (Estados Unidos) se calculó una incidencia de 125 por 100.000 habitantes para el periodo 1976 a 1980; en California fue de 115 por 100.000 en 1988; en Bucaramanga (Colombia) se observó una prevalencia de $15.8 \%$ de los odontólogos evaluados. ${ }^{3-5}$

Se ha establecido que el compromiso sistémico puede ser un factor determinante en la aparición del STC, el uso de instrumental rotatorio por tiempo superior a 240 minutos al día, lo que impide a estos odontólogos trabajar por tiempos prolongados..$^{6-8}$

Por lo anteriormente expuesto, se hace necesario la creación de un programa orientado a prevenir el STC en los estudiantes de odontología, con el fin de evaluar sus conocimientos sobre el tema, los factores de riesgo y la formar de prevenir el STC para aumentar su comprensión y evitar la aparición de este síndrome en esta población.

\section{MATERIALES Y MÉTODOS}

Se realizó un estudio experimental del tipo ensayo clínico controlado en el cual se recolectó información relacionada con el Síndrome del Túnel Carpiano (STC) antes y después de la intervención educativa.

El universo estuvo constituido por 539 estudiantes de la Facultad de Odontología de I a X semestre de la Universidad Santo Tomás, matriculados en el primer periodo académico del 2007. La muestra fue integrada por 53 estudiantes no intervenidos y 52 estudiantes intervenidos para un total de 105 personas. Se consideraron como criterios de inclusión: estudiantes de odontología de la Universidad Santo Tomás matriculados a partir del 2007. Como criterios de exclusión: estudiantes de odontología que no estuvieron presentes en el momento de realizar la encuesta y que no quisieran participar en el estudio.

Se realizó el muestreo no probabilístico por cuotas para un total de 105 estudiantes, conformados por: 10 estudiantes en el primer, segundo, tercer, quinto, sexto, séptimo, y noveno semestre, 11 estudiantes en el cuarto semestre, 13 estudiantes en el octavo semestre, y 11 estudiantes en el decimo semestre.

Se identificaron las variables y se recolectaron en un instrumento; se realizó la prueba piloto en 20 estudiantes de odontología, se efectuaron los procedimientos necesarios con el fin de verificar instrumentos, procedimientos y el tiempo requerido en el proceso investigativo.

Se aplicó la primera encuesta que interrogó sobre los conocimientos, factores de riesgo, factores preventivos del STC y selección el medio de difusión para el material educativo. Se realizó la intervención a los estudiantes que salieron seleccionados mediante asignación a grupo intervención y control en forma aleatoria para cada semestre.

La intervención educativa consistió en la realización de una charla didáctica, un video que contenía explicación de los ejercicios con la fisioterapeuta y una charla sobre la relación del STC con la odontología; se resolvieron las dudas y se procedió a realizar la segunda encuesta después de la intervención, en un tiempo aproximado de 30 minutos. 
Se hizo la codificación, la digitación de duplicados de los datos en Excel y verificación de la calidad de la digitación a través de la rutina Valídate, se corrigieron las inconsistencias se exportaron a Stata 9.0 y se aplicaron las pruebas estadísticas correspondientes, se procedió a analizar los resultados., ${ }^{9,10}$

El plan de análisis se realizó de la siguiente manera: se describieron cada una de las variables mediante medidas de resumen según la naturaleza y posteriormente, se estableció la relación entre la variable de salida intervención con cada variable cuantitativas como cualitativas explicatorias. Se aplicaron las pruebas estadísticas para variables cuantitativas independiente se aplicó la prueba t de Student o test de rangos de Wilcoxon correspondiente a distribución normal.

Para cada una de las variables cualitativas dependientes e independientes en relación de la variable de salida intervención, se aplicaron la prueba estadística $\mathrm{Chi}^{2}$ o test exacto de Fisher, considerando una significancia de $\mathrm{p}<0.05$.

\section{RESULTADOS}

\section{Variables sociodemográficas.}

Se evaluaron 105 estudiantes, con un promedio de edad de $20.8 \pm 3.5$; el $74.3 \%$ (78) eran mujeres. Del total de estudiantes, el 50.5\% (53) no fueron intervenidos, se registró una diferencia estadísticamente significativa en cuanto a la edad entre los intervenidos y los no intervenidos ya que el promedio de edad de los no intervenidos fue de $19.3 \pm 3.1$ y el promedio de los intervenidos fue de $22.3 \pm 3.3$; del total de mujeres encuestadas, el $50 \%$ (39) no fueron intervenidas, del total de hombres encuestados el 51\% (14) no fueron intervenidos.

En cuanto a la procedencia, el 41.5\% (17) de los intervenidos eran de Bucaramanga y el $54.7 \%$ (35) eran de una procedencia diferente. Del total de estudiantes no intervenidos, el 18.9\% (10) eran de primer semestre, el mismo porcentaje se observó para segundo, tercero y séptimo semestre, un porcentaje del $20.7 \%$ (11) eran para cuarto semestre, un $3.7 \%$ (2) pertenecían a sexto semestre; para el total de estudiantes intervenidos, el $19.2 \%$ (10) eran de quinto semestre, un porcentaje igual para noveno semestre, $15.4 \%$ (8) eran estudiantes de sexto semestre, $25 \%$ (13) eran estudiantes de octavo semestre y un porcentaje de $21.1 \%$ (11) eran estudiantes de décimo semestre (Tabla 1).

\section{Variables de conocimiento antes y después de la intervención.}

Del total de estudiantes evaluados sobre el nivel de conocimiento del STC antes de la intervención, se obtuvo un nivel de conocimiento medio de $48.4 \%$ (31). Del total de estudiantes evaluados sobre el nivel de conocimiento de STC después de la intervención, se obtuvo un $82 \%$ (41) nivel de conocimiento alto y un $74.4 \%$ (32) de los no intervenidos un nivel de conocimiento medio.

En el nivel de conocimiento sobre los factores de riesgo del STC antes de la intervención, el $64.1 \%$ (25) en el grupo no intervenidos obtuvieron un nivel de conocimiento bajo y después de la intervención el 76.9\% (40) obtuvieron un nivel de conocimiento alto. El $72.7 \%$ (32) de los no intervenidos obtuvieron un nivel de conocimiento medio.

Del total de estudiantes evaluados sobre la prevención de STC antes de la intervención, el $55.8 \%$ (24) del grupo no intervenido obtuvieron un nivel de conocimiento bajo y después de la intervención el 76.9\% (40) obtuvieron un nivel de conocimiento alto; el $72.7 \%$ (32) de los no intervenidos obtuvieron un nivel de conocimiento medio.

Del total de estudiantes, en el consolidado de respuestas del instrumento antes de la intervención, el $71.9 \%$ (23) del grupo control obtuvieron un nivel de conocimiento bajo y el 59.4\% (41) del grupo intervenido obtuvieron un nivel de conocimiento medio. En el consolidado de respuestas del instrumento después de la intervención, el 83\% (43) obtuvieron un nivel de conocimiento alto; el $83 \%$ (44) del grupo control, obtuvo un nivel de conocimiento medio en (Tabla 2).

Para la selección del elemento como material educativo, el $1.9 \%$ (2) no seleccionaron elemento educativo, el $25.7 \%$ (27) seleccionaron folletos, el 9.5\% (10) escogieron cartillas, el 57.1\% (60) y el 5.7\% (6) escogieron la opción "otro." El $48.3 \%$ (29) del total del grupo no intervenido y el 51.7\% (31) del grupo intervenido seleccionaron la opción charla (Tabla 3).

En relación con el agrado frente a la charla y el video, según los criterios de evaluación y las respuestas de los estudiantes intervenidos, se observó que el 82.75 (43) lo calificó como bueno y el $17.3 \%$ de los estudiantes respondieron que debían mejorar algunos aspectos de la calidad del material educativo. El 5.8\% (3) expresó que debían mejorar las distribución de las imágenes, el 3.8\% (2) el color del fondo fue agradable y el $1.9 \%$ (1) expresó que se debía mejorar el tipo de letra, la metodología del grupo, la metodología utilizada en el medio de difusión, la redacción, el diseño y la ortografía utilizada (Tabla 4). 
Tabla 1. Descripción de las variables sociodemográficas según intervención en los estudiantes de odontología.

\begin{tabular}{|c|c|c|c|c|}
\hline \multirow{2}{*}{ VARIABLE } & \multirow{2}{*}{$\begin{array}{c}\text { GLOBAL } \\
n(\%)\end{array}$} & \multicolumn{2}{|c|}{ INTERVENCIÓN } & \multirow{2}{*}{$\mathbf{P}$} \\
\hline & & NO $(n=53)$ & SI $(n=52)$ & \\
\hline $\begin{array}{l}\text { Edad (años) } \\
\text { Género }\end{array}$ & $20.8 \pm 3.5$ & $19.3 \pm 3.1$ & $22.3 \pm 3.3$ & $\begin{array}{c}{ }^{* *}<0.0001 \\
{ }^{*} 0.868\end{array}$ \\
\hline 0 femenino & $78(74.3)$ & $39(50)$ & $39(50)$ & \\
\hline 1 masculino & $27(25.7)$ & $14(51)$ & 48 (49) & \\
\hline Procedencia & & & & ${ }^{*} 0.186$ \\
\hline 1 Bucaramanga & 41 (39.1) & 24 (58.5) & 17 (41.5) & \\
\hline 2 Otro lugar & $64(60.9)$ & 29 (45.3) & $35(54.7)$ & \\
\hline Semestre & & & & ${ }^{*}<0.0001$ \\
\hline 1 Primero & $10(9.52)$ & 10 (18.9) & --- & \\
\hline 2 Segundo & $10(9.52)$ & 10 (18.9) & --- & \\
\hline 3 Tercero & $10(9.52)$ & $10(18.9)$ & --- & \\
\hline 4 Cuarto & $11(10.5)$ & $11(20.7)$ & --- & \\
\hline 5 Quinto & $10(9.52)$ & --- & $10(19.23)$ & \\
\hline 6 Sexto & $10(9.52)$ & $2(3.7)$ & $8(15.4)$ & \\
\hline 7 Séptimo & $10(9.52)$ & 10 (18.9) & --- & \\
\hline 8 Octavo & $13(12.4)$ & --- & $13(25)$ & \\
\hline 9 Noveno & $10(9.52)$ & --- & $10(19.2)$ & \\
\hline 10 Décimo & $11(10.5)$ & --- & $11(21.1)$ & \\
\hline
\end{tabular}

Tabla 2. Descripción de las variables de conocimiento analizadas en forma global y según intervención en los estudiantes de la Universidad Santo Tomás.

\begin{tabular}{|c|c|c|c|c|}
\hline \multirow{2}{*}{ VARIABLE } & \multirow{2}{*}{$\begin{array}{c}\text { GLOBAL } \\
n(\%)\end{array}$} & \multicolumn{2}{|c|}{ INTERVENCIÓN } & \multirow{2}{*}{$\mathbf{P}$} \\
\hline & & NO $(n=53)$ & SI $(\mathrm{n}=52)$ & \\
\hline Nivel de conocimiento del STC ANTES de la intervención & & & & ${ }^{*} 0.355$ \\
\hline Bajo & $30(28.6))$ & $18(60.0)$ & $12(40.0)$ & \\
\hline Medio & $64(60.9)$ & $31(48.4)$ & $33(51.6)$ & \\
\hline Alto & $11(10.5)$ & $4(36.4)$ & $7(63.6)$ & \\
\hline Nivel de conocimiento del STC DESPUÉS de la intervención & & & & ${ }^{*} 0.0001$ \\
\hline Bajo & $12(11.4)$ & $12(100.0)$ & --- & \\
\hline Medio & $43(40.9)$ & $32(74.4)$ & $11(25.6)$ & \\
\hline Alto & $50(47.6)$ & $9(18.0)$ & $41(82.0)$ & \\
\hline $\begin{array}{l}\text { Nivel de conocimiento de factores de riesgo del STC ANTES de la } \\
\text { intervención }\end{array}$ & & & & ${ }^{*} 0.088$ \\
\hline Bajo & $39(39.1)$ & $25(64.1)$ & $14(35.9)$ & \\
\hline Medio & $47(44.8)$ & $19(40.4)$ & $28(59.6)$ & \\
\hline Alto & 18 (18.1) & $9(47.4)$ & $10(52.6)$ & \\
\hline $\begin{array}{l}\text { Nivel de conocimiento de factores de riesgo del STC DESPUÉS de la } \\
\text { intervención }\end{array}$ & & & & $*<0.0001$ \\
\hline Bajo & $9(8.6)$ & $9(100.0)$ & --- & \\
\hline Medio & 44 (41.9) & $32(72.7)$ & $12(27.3)$ & \\
\hline Alto & $52(49.5)$ & $12(23.1)$ & $40(76.9)$ & \\
\hline $\begin{array}{l}\text { Nivel de conocimiento sobre la prevención del STC ANTES de la } \\
\text { intervención }\end{array}$ & & & & ${ }^{*} 0.611$ \\
\hline Bajo & $43(40.9)$ & $24(55.8)$ & $19(44.2)$ & \\
\hline Medio & 50 (47.6) & $24(48.0)$ & $26(52.0)$ & \\
\hline Alto & $12(11.4)$ & $5(41.7)$ & $7(58.3)$ & \\
\hline $\begin{array}{l}\text { Nivel de conocimiento sobre la prevención del STC DESPUÉS de la } \\
\text { intervención }\end{array}$ & & & & $*<0.0001$ \\
\hline Bajo & $9(8.6)$ & $9(100.0)$ & --- & \\
\hline Medio & 44 (41.9) & $32(72.7)$ & $12(27.3)$ & \\
\hline Alto & $52(49.5)$ & $12(23.1)$ & $40(76.9)$ & \\
\hline $\begin{array}{l}\text { Consolidado de respuestas sobre el conocimiento del STC ANTES de la } \\
\text { intervención }\end{array}$ & & & & ${ }^{*} 0.0014$ \\
\hline ( & $32(30.5))$ & 23 (71.9) & $9(28.1)$ & \\
\hline Medio & $69(65.7)$ & $28(40.6)$ & $41(59.4)$ & \\
\hline & $4(3.8)$ & $2(50.0)$ & $2(50.0)$ & \\
\hline $\begin{array}{l}\text { Consolidado de respuestas sobre el conocimiento del STC DESPUES de la } \\
\text { intervención }\end{array}$ & & & & ${ }^{*}<0.0001$ \\
\hline Bajo & $9(8.6)$ & $9(17.0)$ & --- & \\
\hline Medio & $53(50.5)$ & $44(83.0)$ & 9 (17.0) & \\
\hline Alto & $43(40.9)$ & --- & $43(83.0)$ & \\
\hline
\end{tabular}


Tabla 3. Descripción de la preferencia del elemento seleccionado por el sujeto evaluado durante la primera encuesta.

\begin{tabular}{lcccc}
\hline \multirow{2}{*}{\multicolumn{1}{c}{ VARIABLE }} & GLOBAL & \multicolumn{2}{c}{ INTERVENCIÓN } & \multirow{2}{*}{ P } \\
\cline { 2 - 4 } Medio de difusión & $\boldsymbol{n}(\%)$ & NO & SI & \\
0, No selecciono & $2(1.9)$ & $2(100.0)$ & & \\
1, Folleto & $27(25.7)$ & $13(48.2)$ & $14(51.8)$ & \\
2, Cartilla & $10(9.5)$ & $8(80.0)$ & $2(20.0)$ \\
3, Charla & $60(57.1)$ & $29(48.3)$ & $31(51.7)$ \\
4, Otros & $6(5.7)$ & $1(16.7)$ & $5(83.3)$ & \\
\hline
\end{tabular}

${ }^{*}$ chi $^{2} \quad{ }^{*}$ Test exacto de Fisher $\alpha=0.05$

Tabla 4. Agrado frente a los criterios de evaluación del medio de difusión escogido, analizado en forma global y según intervención.

\begin{tabular}{lcc}
\hline \multicolumn{1}{c}{ VARIABLE } & \multicolumn{2}{c}{ RESPUESTA } \\
\cline { 2 - 3 } & \multicolumn{2}{c}{ NO } \\
\hline Agrado frente a la charla y medio de difusión según los criterios analizados & $9(17.3)$ & $43(82.7)$ \\
Tipo de letra agradable & $1(1.9)$ & $51(98.1)$ \\
Color agradable & $2(3.8)$ & $50(96.2)$ \\
Metodología del grupo & $1(1.92)$ & $51(98.1)$ \\
Metodología utilizada en el medio de difusión & $1(1.92)$ & $51(98.1)$ \\
Distribución de las imágenes & $3(5.8)$ & $49(94.2)$ \\
Distribución del tema & -- & $52(100.0)$ \\
Redacción, diseño, ortografía. & $1(1.92)$ & $51(98.1)$ \\
\hline
\end{tabular}

\section{DISCUSIÓN}

En la literatura revisada se han encontrado pocos reportes de programas orientados a prevenir el Síndrome del Túnel Carpiano en los estudiantes de odontología; se observó que la mayoría de estos programas se han realizado en trabajadores de empresas avícolas y operarios de costura. ${ }^{8}$

En un estudio en odontólogos de la ciudad de Bucaramanga realizado en la Universidad Santo Tomas, se demostró una la prevalencia del Síndrome del Túnel Carpiano en el $15.8 \%$ de la población evaluada, ${ }^{4}$ esta preocupación condujo a la necesidad de formular un programa de promoción y prevención con estrategias de aprendizaje que evalúen el grado de conocimiento sobre la prevención y los factores de riesgo del STC. ${ }^{6}$

Se desarrolló un programa orientado a prevenir el STC en estudiantes de odontología de I a X de la Universidad Santo Tomás, el cual se inició evaluando el nivel de conocimiento antes de la intervención, tal como plantea Navarro y colaboradores como requisito elemental para la realización de este tipo de programas. ${ }^{6}$

Los resultados que proyectaron las encuestas preliminares del estudio indicaron un nivel de conocimiento medio de los estudiantes; luego de la aplicación del programa educativo en el grupo intervenido se observaron cambios estadísticamente significativos en el consolidado de las respuestas sobre el STC, se obtuvo un nivel de conocimiento alto en el $83 \%$ de los intervenidos, mientras que el grupo control obtuvo un $83 \%$ de nivel de conocimiento medio.

Los resultados obtenidos con la intervención demuestran que es una buena estrategia comunicativa y preventiva implementar programas orientados a prevenir el STC en estudiantes de odontología, para lograr un impacto a corto plazo, lo que coincide con el estudio de Navarro y colaboradores, el cual muestra que la aplicación del programa preventivo con material educativo multimedia, aumentó de manera favorable el nivel de conocimiento sobre el STC y mejoró las posibilidades de aprendizaje en el estudiante, además de generar inquietudes frente al tema. ${ }^{6}$

Verhagen y colaboradores, en un estudio a largo plazo en el 2003, determinaron pruebas limitadas sobre la efectividad de ejercicios con STC. ${ }^{11}$ Observaron un eficaz abordaje individual en comparación con un abordaje grupal, resultados contrarios al presente programa en el cual se intervino de forma grupal y se obtuvo alta efectividad de los ejercicios debido a que aumentó el nivel de conocimiento sobre la prevención del STC en el 76\% de los estudiantes. Coincide con lo expresado con O'connor y colaboradores quienes demostraron que la prevención del STC a través de ejercicios de estiramiento antes que se desarrolle la enfermedad tiene una alta efectividad en comparación con su realización después de la aparición de los síntomas. ${ }^{12}$ 
Según Silverstein, las modificaciones en el trabajo podrían disminuir la prevalencia o incidencia del STC laboral. ${ }^{13}$ La prevención primaria debe basarse en la adaptación de los instrumentos de trabajo, en la instauración de programas ergonómicos como ejercicios preventivos, tal como lo expresa Montgomery "el bienestar del cuerpo empieza con aprender a cuidar de él y para esto es necesario asistir a programas diarios de ejercicios, masajes, terapia fisica, nutrición y otros métodos para disminuir la tensión muscular y ayudar a prevenir síndrome del túnel carpiano y otros desordenes músculo-esqueléticos que pueden alterar la calidad de vida del personal odontológico y del estudiante". ${ }^{5}$

En la revisión de la literatura, se observó el interés por el estudio del síndrome del túnel carpiano en varios aspectos como lo es su diagnostico y tratamiento con fármacos, cirugías o con fisioterapia. Sin embargo, hay pocos estudios dirigidos a programas de prevención del STC, y en los que se han realizado se observa que la mayor parte de los participantes son mujeres, igual que en el presente estudio, lo cual es favorable para este género pues se ha demostrado que el STC tiene doble probabilidad de desarrollarse en mujeres debido a la exposición de múltiples factores de riesgo.

McDiarmid reportó que en el STC el problema es el trabajo y no el genero, con lo que pone en contradicción la contribución de factores como el nivel hormonal, la antropometría u otras características asociadas al género femenino, muchas veces usadas para explicar la mayor frecuencia de aparición del STC en las mujeres. Este enfoque podría retrasar el establecimiento de medidas preventivas laborales y aplazar la oportunidad de reducir los daños laborales en la salud de las mujeres. ${ }^{14}$

Es importante resaltar que este es el único estudio reportado en la ciudad de Bucaramanga en donde se realiza un programa orientado a prevenir el STC en estudiantes de odontología. Esto podría considerarse como una fortaleza. El Ministerio de Educación Nacional, en su Decreto 2566 de septiembre 10 de 2003, hace énfasis en la utilización del tiempo en actividades independientes, por lo que los materiales educativos multimedia se convierten en una herramienta indispensable.

El capitulo VI de la Ley 1122 del 2007 del Ministerio de Protección Social mediante las políticas de salud publica expresa los objetivos correspondientes a la atención y prevención de los principales factores de riesgo para la salud y promoción de condiciones y estilos de vida saludables, siendo también una de las funciones de las administradoras de riesgos profesionales. ${ }^{15}$
De igual manera, en el consejo de Bogotá se encuentra radicado un proyecto por medio del cual se institucionaliza una semana de prevención del STC en cumplimiento con el Decreto 1832 de 1994 y el Decreto 1295 del Sistema General de Riesgos Profesionales. ${ }^{16,17}$

Un programa de educación del Síndrome del Túnel Carpiano, por medio de ejercicios, ayuda a prevenir su aparición y sus consecuencias tanto personales como económicas, pues la importancia del diagnóstico precoz evita tratamientos con cirugías costosas y dolorosas, y con la posibilidad de tratamientos más rápidos y menos agresivos. ${ }^{12,18}$

\section{CONCLUSIONES}

Se recomienda realizar más programas educativos e investigar más estrategias para prevenir el Síndrome del Túnel Carpiano por parte de la universidad, para crear más conciencia sobre la realidad de esta enfermedad en los estudiantes de odontología y también en los docentes de la Facultad; al mismo tiempo, es necesario realizar jornadas de ejercicios preventivos en los estudiantes de preclínica y clínica, en las auxiliares de odontología y en el personal expuesto a los factores de riesgo del Síndrome del Túnel Carpiano.

\section{BIBLIOGRAFÍA}

1. Silverstein B, Fine LJ, Armstrong TJ. Occupational factors and carpal tunnel syndrome. Am J Ind Med 1987; 11: 343 - 358.

2. Hernández D, Castro R, Wolff AG, Raigoza LI. Rehabilitación en lesiones de nervios periféricos de antebrazo y mano. Proyecto ISS-ASCOFAME 2006.

3. Lalumandier JA, McPhee SD. Prevalence and risk factors of hand problems and carpal tunnel syndrome among dental hygienists. J Dent Hyg 2001; 75: 130 - 134.

4. Rodríguez GA, Morales K, Maldonado L, Jiménez G, Quiroz LF, Concha SC, Rueda SJ. Prevalencia del síndrome de túnel carpiano y sus factores asociados en odontólogos de la ciudad de Bucaramanga y de su área metropolitana. Ustasalud Odontología 2005; 4: 73 - 80.

5. Montgomery K. Preventing Carpal Tunnel Syndrome and other upper-body musculoskeletal injuries. Clinical Feature 2003.

6. Navarro H, Puentes L, Sayago L, Sayago H. Material educativo en prevención de patologías producidas por mal posición de trabajo en odontología. [Trabajo de Grado]. Bucaramanga: Universidad Santo Tomás; 2006.

7. Roel-Valdés J, Arizo-Luque V, Ronda-Pérez E. Epidemiología del síndrome del túnel carpiano de origen laboral en la provincia de Alicante. Rev Esp Salud Pública [en línea] 2006; [fecha de acceso: septiembre de 2006] 89: 395 - 409. URL disponible en: http://www.msc.es/ca/ biblioPublic/publicaciones/recursos_propios/resp/revista_cdrom/vol80/vol80_4/RS804C_395.pdf

8. Gutiérrez CM, Cotes ML. Programa fisioterapéutico para prevenir el síndrome del túnel carpiano en operarios de costura. Fundación Universidad Manuela Beltrán. Umbral científico. P. 2032, Bogotá, Colombia 2006. 
9. CDC-OMS. Epi-Info 6 Versión 6.04a , Noviembre de 1996.

10. Microsoft Excel, 5.0, Microsoft Corporation, 1997.

11. Verhagen AP, Karels C, Bierma-Zeinstra SMA, Burdorf L, Feleus A, Dahaghin S, de Vet HCW, Koes BW. Intervenciones ergonómicas y fisioterapéuticas para el tratamiento de las molestias relacionadas con trabajo en el brazo, cuello u hombro en adultos. La Biblioteca Cochrane Plus [resumen en línea] 2006. URL disponible en: http://www.cochrane.org/reviews/es/ ab003471.html

12. O'Connor D, Marchall S, Massy N. Tratamiento no quirúrgico para el síndrome del túnel carpiano. La Biblioteca Cochrane Plus [resumen en línea] 2003.

13. Silverstein BA, Fine LJ. Cumulative trauma disorders of the upper extremity. A preventive strategy is needed. J Occupational Med 1991; 33: 642 - 644.

14. McDiarmid M, Oliver M, Ruser J, Gucer P. Male and female rate differences in carpal tunnel syndrome injuries: personal attributes or job tasks? Environ Res 2000; 83: $23-32$.

15. República de Colombia. Ministerio de Protección Social. Ley 1122 del 2007. Bogotá Colombia, 9 de enero del 2007.

16. Medina Y. Proyecto de prevención del Síndrome Del túnel carpiano acuerdo N. 294 de 2006. Concejo de Bogotá- Colombia.

17. República de Colombia. Ministerio de Salud. Decreto 1832 de 1994 y el Decreto 1295 del sistema general de riesgos profesionales.

18. Macfarlane GJ. Identification and prevention of workrelated carpal tunnel syndrome. Lancet 2001; 357: 1146 $-1147$.

19. Mohammed A, Harding MG. Carpal tunnel syndrome in haemodialysis patients: early detection by electroneurophysiological. Studies nephrology 2001; 6: $259-262$.
Universidad Santo Tomas

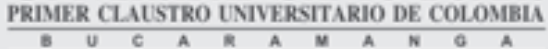
iHace Pais!

\section{Especialización en} Odontopediatría

\section{ODONTOLOGÍA INFANTIL}

\section{Servicios de Odontopediatría y Ortodoncia Preventiva}

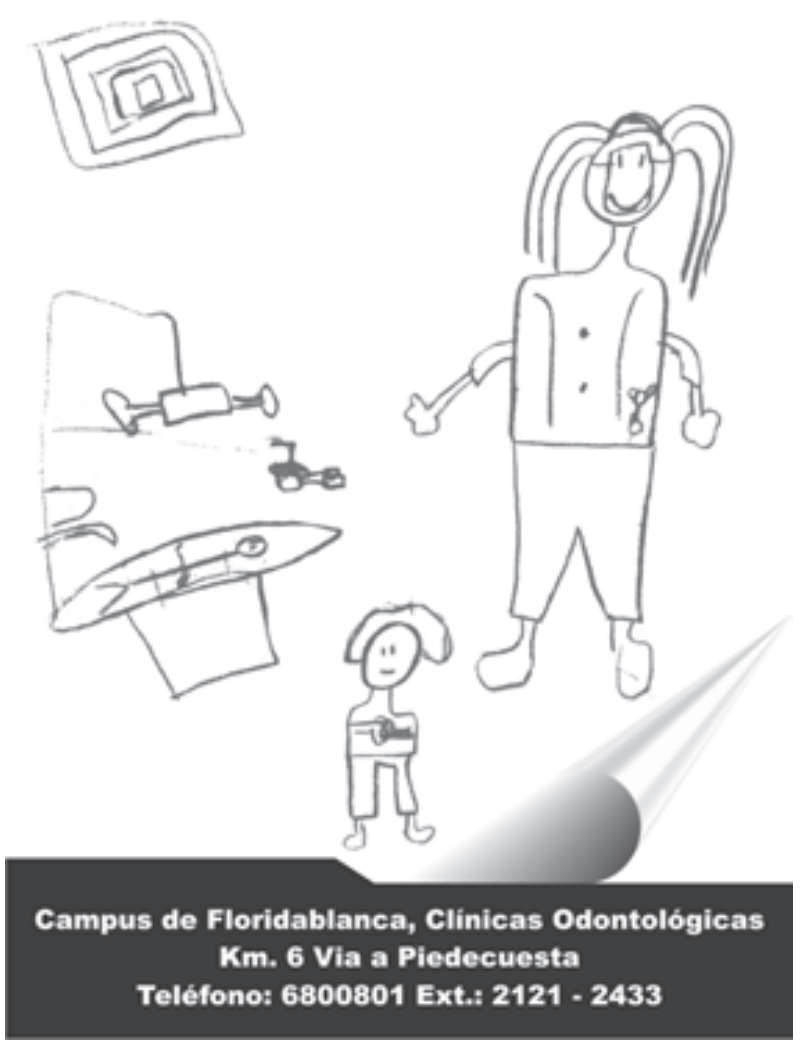

